\title{
An Approach for Personalized Web-based Vocabulary Learning through Word Association Games*
}

\author{
Jaeyoung Jung \\ Human System Science \\ Tokyo Institute of Technology, Japan \\ catherina@dp.hum.titech.ac.jp
}

\author{
Sabine Graf \\ Graduate Institute of Learning \& Instruction, \\ National Central University, Taiwan \\ sabine.graf@ieee.org
}

\begin{abstract}
Learning vocabularies is an essential task when learning a foreign language. However, most learners seem to experience similar difficulties in learning vocabularies in terms of large burden and a lack of motivation. In this paper, we propose an approach for web-based vocabulary learning through personalized word association games. By applying game-based learning, we aim at making vocabulary learning more attractive and more fun for learners. On the other hand, personalized learning has high potential to make vocabulary learning more effective by suiting the learning process to the students' individual needs. The paper shows how these two learning concepts can be integrated in an educational system, demonstrating the architecture and functionality of a personalized, gamebased vocabulary learning system.
\end{abstract}

\section{Introduction}

Computer-assisted language learning (CALL) provides learners with easy access to learning environments irrespective of place and time, and increases motivation and effectiveness of learning with multimedia content. Furthermore, many efforts have already been made for overcoming some shortages often observed in CALL, such as flexible interaction, adaptivity, and personalization. Some CALL systems, e.g., ICICLE [1] and E-tutor [2], show such improvements by using student modeling techniques for correcting grammatical errors and giving feedbacks based on learners' production and according to learners' level of proficiency.

\footnotetext{
${ }^{*}$ This research has been funded by the Grants-in-Aid for Scientific Research of Japan Society for the Promotion of Science and the National Science Council of the Republic of China, Taiwan, under Contract No. NSC 097-2811-S-008-001-.
}

The importance of vocabulary in language learning has led active studies on vocabulary acquisition theory and vocabulary learning strategies. The considerable attention has produced various CALL systems for vocabulary learning, such as CAVOCA [3], WUFUN [4], and PowerWords [5]. Rather than simply supporting a self-study for vocabulary learning, these lexical CALL systems provide learners with various types of learning tasks, based on the learners' vocabulary levels and the learning history. However, in fact many CALL systems still have a tendency of dealing with vocabulary learning as a simple complement to a reading skill. Therefore, the need arises towards systems that fully focus on supporting learners in vocabulary learning and providing them with a learning environment that adapts to their individual knowledge, preferences, and needs as well as motivates and encourages students to learn vocabularies. Thus, more vigorous studies need to be conducted on CALL for vocabulary learning in order to provide interaction, personalization, and motivation, complying with the learner's need.

In this sense, this paper proposes an approach for a web-based vocabulary learning system that is designed based on two key learning concepts, personalized learning and game-based learning, which have been paid increasing attention to in e-learning in the last years. As Brusilovsky [6] describes, adaptivity and personalization in educational systems have high potential to help learners in learning by, for example, fitting the content to their needs or providing them with adaptive navigation support. Furthermore, Burgos et al. [7] and Amory [8] point out that educational electronic games can provide pedagogical elements such as interactivity, problem solving, user involvement, motivation, and creativity. In addition, adaptive and personalized aspects can be incorporated in games [9].

This paper presents an approach for personalized and game-based vocabulary learning, aiming at making 
vocabulary learning more interesting, more effective, and easier for learners. Section 2 describes the details of our approach. Subsequently, the architecture of a system that incorporates the strategies of personalized and game-based vocabulary training is presented. In Section 4, we explain the functionality and the workflow of the system in more detail. Section 5 concludes the paper.

\section{A new approach for vocabulary learning}

Learning by means of associations is one of the well-known learning strategies. Crow and Quigley [10] show the effectiveness of this strategy through empirical evidence. In this study, we apply the concept of associations in order to compose a game for vocabulary learning. Instead of memorizing given associated pairs, learners are encouraged to produce their own associations or find out possible associative relationships through game-based tasks. During this process, learners are expected to spontaneously practice to repeatedly recall the new words and to make meaningful connections between their prior lexical knowledge and the new words.

Another key concept in the proposed approach is personalized (or adaptive) learning which means that the content and learning material is adapted to the learners' needs. Many different characteristics and needs of learners can be considered in an adaptive learning system. An adaptive vocabulary learning system is conceivable in the sense that the knowledge in terms of known words, the learners' preferred learning styles, and their cognitive abilities can be incorporated when learning new vocabularies, making the process of learning easier for the learners. Jonassen and Grabowski [11] summarized that prior knowledge is one of the strongest and consistent individual difference predictors of achievement, which makes it an important factor for adaptation and personalization in learning environments. As a consequence, the personalization strategy in the proposed approach focuses on considering the learners' knowledge in terms of known vocabularies and uses this information for providing learners with personalized vocabulary training.

\section{Architecture of a personalized and game-based vocabulary learning system}

Adaptive and personalized educational systems are designed for providing different learning experience for different learners, considering the individual needs and characteristics. While there exist several models and frameworks for adaptive systems (e.g., [12], [13]), we based our architecture on a model proposed for intelligent educational (or tutoring) systems [14], which emphasizes the educational aspects. Accordingly, 4 modules are necessary to provide personalized contents. The expert module is responsible for the domain knowledge, which is stored in the expert model and for the internal representation of the domain knowledge in the system. The student module deals with gathering relevant data about the learners and storing them in the student model. The tutoring module concerns with the determination of learning materials depending on the learners' needs, and the interface module deals with presenting those contents. The proposed system is constituted of these 4 components (Fig. 1), each of which is specified in the following subsections.

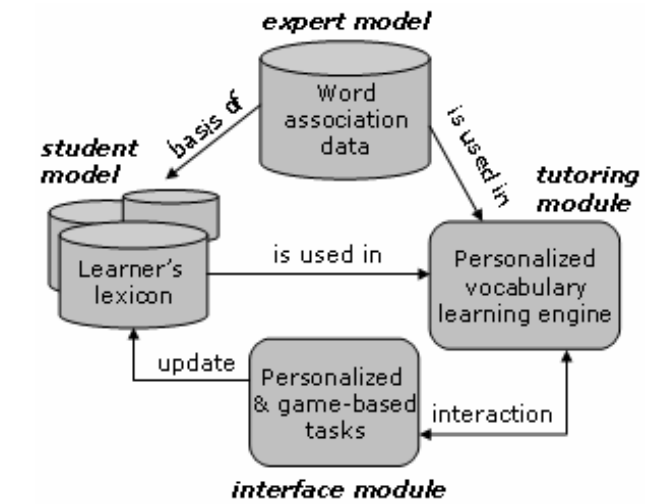

Figure 1. Architecture of a personalized vocabulary learning system

\subsection{Word association data (expert module)}

For constructing a database including vocabularies and associative information, word association data are used, such as Nelson's word association [15] and Edinburgh Word Association Thesaurus [16]. The database is built in the form of a semantic network, where words are represented by nodes and the associative relations of words are described by edges. The semantic network graph is additionally clustered by graph clustering methods, and is reorganized into two different types, that is, conceptual grouping and associative grouping [17]. Eventually, 3 different types of semantic networks are included in the database: the original word-basis graph and the two types of clusterbasis graphs. From each of these networks, different types of associative information can be extracted.

\subsection{Learners' lexicon (student module)}

Similarly to the expert model, the learners' lexical knowledge is represented as a network graph, taking 
the learners' words as nodes and the associative relationships of words as edges. The learners' lexicon collected in the student model represents the system's view about the learners' pre-existing lexical knowledge. Therefore, the student model grows as the learners keep conducting the learning tasks and learning new words. In turn, the data in the student model is accessed and used for composing the suitable learning tasks for each of the learners based on their knowledge.

\subsection{Personalized vocabulary engine (tutoring module)}

The personalized vocabulary engine is responsible for calculating and controlling the tasks during a learning session. This includes making decisions about which words should be presented to obtain word associations and also which information should be used to help the learning of new words, taking into account the learners' prior knowledge of vocabulary.

\subsection{Personalized tasks (interface module)}

The interface module is responsible for the GUIs. It is concerned in effectively presenting the learning materials that are generated by the tutoring module and in the easy and friendly interaction with learners.

\section{Functionality \& workflow of the system}

A learning session is composed of 5 stages. This procedural division indicates how the personalized vocabulary engine works under the interface module, in order to generate adaptive tasks for each learner. To the learners, the tasks may seem like small pieces of one complete game. In the following sections, the 5 stages are described.

\subsection{Initial data setting}

The first stage of the learning session aims at initializing and enlarging the information about the learner's pre-existing lexicon. For this, a game-based task is conducted by asking the learners to produce as many associations as possible within a certain time. In this task, one word is presented, which is randomly chosen by the system. If this word is unknown by the learner, he/she can ask for another word. In this case, the system provides the learner with a likely easier word than the previous one by selecting a word which is more frequently used. The usage of words is calculated by the tutoring module based on the number of associations that a word has to other words, assuming that the number of associations reflect the frequency of its usage.

\subsection{Determining the target words}

In stage two, the candidate target words (around 10 words) are selected. These words are the focus of learning during the session. The personalized vocabulary engine extracts the words from the expert model, choosing only words which do not overlap with the words stored in the student model. However, the student model represents only a subset of the learner's mental lexicon, and therefore, the candidate target words possibly include some words the learners are familiar with. Therefore, the candidate word list is divided into the known and the new words by the learner's judgment. Both word lists are used for the subsequent games in stage four, but the ones checked as the new words are more focused.

\subsection{Learning the meaning of the target words}

In stage three, the learners acquire the basic meanings of the target words. They are expected to equip themselves with a new lexical knowledge of the target words in this stage, before going to the next stage. By utilizing a free online dictionary, the definition of the words, images, or learner's native language translation are presented. There is no time setting in this stage.

\subsection{Encoding through word association games}

The learners play 3 different types of word association games in stage four, where they are expected to use the lexical knowledge together with their associative ability. The words occurring in this stage are a mixture of familiar and new words, consisting of the words collected through free association in stage one and the target words selected in stage two. From the various associative contexts of the words, the learners repeatedly practice the new words and easily encode them. The three types of games are presented in the following and an example of each game is illustrated in Fig. 2.

(1) Turn-taking: The learners produce associations with the presented word, taking a turn with the system. A time limit is set, so if the learners cannot give the association in a given time, the system takes its turn. At the end, the learners get a score for their performance.

(2) Guessing: Learners are asked to find a word within a given time that can be associated in common with some given words. Some hints are available, by 
showing the number of letters or a part of the word, however the response time and the number of hints is reflected in scoring.

(3) Gap filling: The learners have to find out the concept and subsequently the word to connect two given words. A time limit is set and the system gives hints such as showing some letters.

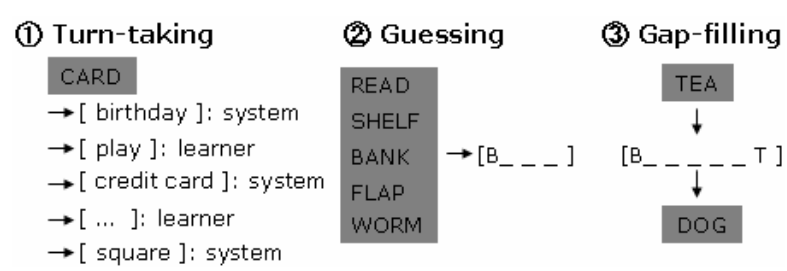

\section{Figure 2. Examples of the 3 different types of word association games}

\subsection{Testing}

In stage five, the learners take a multiple choice test for confirming the newly learnt target words and their basic definition. Only target words for which questions are correctly answered are used for updating the student model.

\section{Conclusion and future work}

This paper addressed personalized vocabulary learning based on association games. In particular, the architecture and functionalities of a system that implements personalized, game-based vocabulary learning was introduced. The combination of the two key concepts of personalized learning and game-based learning aims at supporting more effective and attractive vocabulary learning.

Future work will deal with the implementation of the system based on the proposed approach as well as its evaluation. Another issue for future work is the enhancement of adaptivity in the system, considering learners' learning styles and cognitive abilities in addition to the learners' knowledge.

\section{References}

[1] L.N. Michaud, K.F. McCoy, and L.A. Stark, "Modeling the acquisition of English: An intelligent call approach", in Proc. of the $8^{\text {th }}$ Int'l. Conference on User Modeling, Sonthofen, Germany, 2001.

[2] T. Heift, "Inspectable learner reports for web-based language learning", ReCALL Journal, 16 (2), 2004, pp. 416431.

[3] Peter J.M. Groot, "Computer Assisted Second Language Vocabulary Acquisition", Language Learning \& Technology, vol.4, No.1, 2000, pp. 60-81.
[4] Q. Ma and P. Kelly, "Computer Assisted Vocabulary Learning: Design \& Evaluation", Computer Assisted Language Learning, V19, N1, 2006, pp. 15-45.

[5] C. Kawauchi, "Proficiency Differences in CALLBased Vocabulary Learning: The Effectiveness of Using 'PowerWords'”, FLEAT 5, 2005, pp. 55-65.

[6] P. Brusilovsky, "Methods and Techniques of Adaptive Hypermedia". User Modeling and User-Adapted Interaction, 6 (2-3), 1996, pp. 87-129.

[7] D. Burgos, P. Moreno, J.L. Sierra, B. Fernandez-Manjon, M.Specht, \& R.Koper, "Building Adaptive Game-based Learning Resources: The Marriage of IMS Learning Design and <e-Adventure>", Simulation \& Gaming (in Press), 2008.

[8] A. Amory, "Building an educational adventure game: Theory, design and lessons", Journal of Interactive Learning Research, vol.12, 2001, pp. 249-263.

[9] M. Prensky, Digital Game-based Learning. New York, USA: McGraw-Hill, 2001.

[10] J.T. Crow and J.R. Quigley, "A semantic field approach to passive vocabulary acquisition for reading comprehension", TESOL Quarterly, 19, 1985, pp. 497-513.

[11] D.H. Jonassen and B.L. Grabowski, Handbook of Individual Differences, Learning, and Instruction, Lawrence Erlbaum Associates, Hillsdale, New Jersey, 1993.

[12] P. de Bra, G.-J. Houben, and H. Wu, "AHAM: A DexterBased Reference Model for Adaptive Hypermedia", in Proc. of the ACM Int'l. Conference on Hypertext and Hypermedia, Darmstadt, ACM Press, pp. 147-156.

[13] N. Koch, and M. Wirsing, "The Munich Reference Model for Adaptive Hypermedia Applications", in Proc. of the Int'l. Conference on Adaptive Hypermedia and Adaptive WebBased Systems, Malaga, Spain, Springer, pp. 213-222.

[14] P. Brusilovsky, "The Construction and Application of Student Models in Intelligent Tutoring Systems", Journal of Computer and Systems Sciences Int'l., 32 (1), 1994, pp. 70-89.

[15] D.L. Nelson, C.L. McEvoy, and T.A. Schreiber, The University of South Florida word association, rhyme, and $\begin{array}{lll}\text { word fragment norms, } & 1998\end{array}$ (http://www.usf.edu/FreeAssociation/).

[16] G.R. Kiss, C. Armstrong, R. Milroy, and J. Piper, "An associative thesaurus of English and its computer analysis", in Aitken, A.J., Bailey, R.W. and HamiltonSmith, N. (Eds.), The Computer and Literary Studies. Edinburgh University Press, Edinburgh, 1973.

[17] J. Jung, M. Miyake, and H. Akama, "The Development of a Web-based Free Association Retrieval System Applying Graph Theory", in Proc. of ED-MEDIA, AACE Press, Chesapeake, VA, 2007, pp.3359-3365. 